\title{
The Impact of Oxidative Stress on Dental Implants
}

\author{
César Esquivel-Chirino, Juan Carlos Gómez-Landeros, Erika Patricia Carabantes-Campos, \\ Daniela Carmona-Ruiz, Yolanda Valero-Princet, Christian Márquez-Correa, \\ and José Antonio Morales-González
}

\section{ABSTRACT}

Periodontal disease is an inflammatory condition that alters the periodontium, resulting in destruction of the alveolar bone; without treatment the condition may lead to tooth loss. Dental implants are an alternative for substitution of naturally lost teeth as they have high success rates; however, some factors are related to its failure. Peri-implantitis (PI) is a pathological condition that affects the tissues surrounding dental implants and has been reported as the major cause of implant failure; PI and periodontal diseases are characterized by tissue inflammation and bone damage. In homeostasis conditions, reactive oxygen species (ROS) have been shown to be involved in cell maintenance, signal transduction, and repair of all tissues, but ROS overaccumulation leads to oxidative stress, which generates cell damage and tissue destruction; likewise, antioxidants protect against the destructive effects of ROS by turning free radicals into waste products. The main purpose of this review was to determine some aspects of inflammatory responses and oxidative stress and analyze their relationship with the lack of osseointegration and PI.

Keywords: antioxidants, free radicals, oxidative stress, peri-implantitis.
Published Online: January 19, 2021

ISSN: $2684-4443$

DOI: $10.24018 /$ ejdent.2021.2.1.37

\section{Esquivel-Chirino*}

Area de Básicas Médicas, División de Estudios Profesionales, Facultad de Odontología, Universidad Nacional Autónoma de México, Mexico.

(e-mail: investigaciondental@gmail.com)

J. C. Gómez-Landeros

Especialidad de Periodoncia, Posgrado de la Facultad de Odontología, Universidad Intercontinental, Mexico.

(e-mail: jcglanderos@ ${ }^{@}$ hotmail.com)

E. P. Carabantes-Campos

Especialidad de Periodoncia, Posgrado de la Facultad de Odontología, Universidad Intercontinental, Mexico.

(e-mail: ericarabantes ${ }^{\circledR}$ gmail.com)

D. Carmona-Ruiz

Area de Ortodoncia, División de Estudios Profesionales, Facultad de Odontología, Universidad Nacional Autónoma de México, Mexico.

(e-mail: danyelacarmona@ ${ }^{@ m a i l . c o m) ~}$

Y. Valero-Princet

Facultad de Odontología, División de la Salud, Universidad Intercontinental, Mexico.

(e-mail: mvalero@uic.edu.mx)

C. Márquez-Correa

Coordinador Especialidad de Periodoncia, Posgrado de la Facultad de Odontología, Universidad Intercontinenta, Mexico.

(e-mail: dr.christian.marquez ${ }^{\circledR}$ gmail.com) J. A. Morales-González

Laboratorio de Medicina de Conservación, Escuela Superior de Medicina, Instituto Politécnico Nacional, México.

(e-mail: jmorales101@yahoo.com.mx)

*Corresponding Author

\section{INTRODUCTION}

Dental implants are a proven substitute that can be used to replace naturally lost or hopeless teeth. Their success rates are high, and they can support dental prostheses for prolonged periods of time, up to thirty years [1]; however, there are several factors that can interfere with their osseointegration during the healing phase after its placement. Many factors that could result in short, medium, or long-term complications exist, which can cause subsequent failure of osseointegration. According to the new classification workshop for peri-implant diseases in 2018, these diseases have been classified into peri-implant mucositis (PM) and PI [2].

The term, peri-implantitis (PI), was first introduced in 1987 by Mombelli et al. They established the differences between periodontal and peri-implant diseases (PM and PI, respectively). [3]. PM is an inflammation established at the level of the peri-implant mucosa caused by plaque or poor hygiene [4], and the condition is characterized by bleeding, swelling, and mainly absence of bone loss absence. This condition precedes the development of PI (Fig. 1). In order to 
eliminate PM, dental cleaning procedures are done at the level of perimplant soft tissues. Also important for managing this condition are regimes of plaque control and periodontal health education.

In recent years, various studies have analyzed the incidence, prevalence, and risk factors for PI. In a systematic review covering 36 years, Dreyer et al. identified a prevalence in the general population of $7 \%$. Concerning risk factors, they identified medium and medium-high evidence with respect to development of the illness in patients who are smokers, those without regular maintenance control, and those with diabetes mellitus (DM) or with a history of periodontitis [6]. In their retrospective clinical study by Krebs, it was found that the incidence of PI is $7.9 \%$ at the implant level and $13.2 \%$ at the patient level seventeen years after implant placement [7].

When there is an absence of intimate contact between the implant and the bone before its functional loading after a healing period, we refer to an early failure of the implants, this occurs in $2 \%$ to $6 \%$ of the implants. Lack of osseointegration is not a foreign body reaction but a consequence of bacterial colonization on the implant surface that causes a scar of fibrous tissue to be form around the implant body [1]. In a retrospective study, Krisam et al. included 186 implants placed between the periods from 2012 to 2017 and found nine with failed osseointegration. These failures occurred mainly in short implants, implants placed in previously regenerated areas, implants placed in maxillary areas. Other factors of failed osseointegration that the authors mention is smoking, DM, history of periodontal disease, and poor bone quantity and quality. The finding that there was no relationship between the degrees of torque and the early implant failure is controversial since the lack of primary stability has been considered as a possible cause of absence of osseointegration; also, no relationship between immediate loads and early failures. was found. Healing times to achieve osseointegration in this study were five months for the maxilla and 4.6 months for the maxilla [8].

The aim of this paper was to determine inflammatory and clinical relationships that could lead to PI and the absence of osseointegration in dental implants, providing the perspective of the role that oxidative stress (OS) plays in these conditions. In addition, we will give some clinical suggestions to avoid problems at the different stages of dental implant placement.

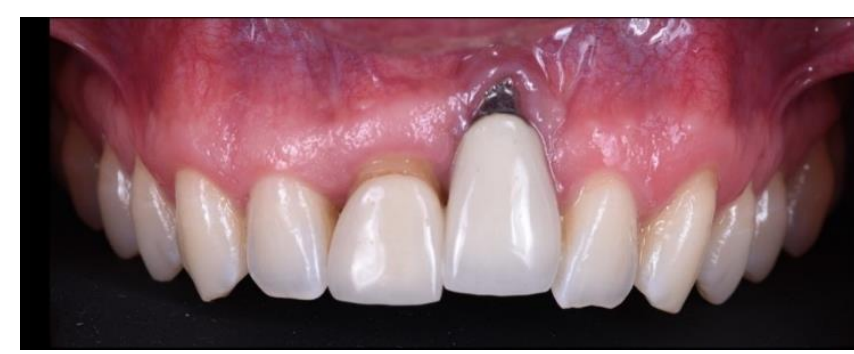

Fig. 1. Peri-implant disease: dental implants placement in a vestibular position that compromises the endosteal circulation and the vestibular bone.

\section{PERI-IMPLANTITIS}

PI is a pathological process that is characterized by inflammation of the tissues surrounding a dental implant that causes a progressive loss of the bone that supports the implant. Known risk factors that may influence their progression include periodontal disease (the main risk factor), poor oral hygiene, bacterial plaque, occlusal overload, diseases such as diabetes mellitus, and smoking. The association with genetic polymorphisms has also been reported. Individuals with poor oral hygiene and smoking have three times greater the probability of suffering PI. [10]. Sahrmann et al reported that an evaluation of 47 studies showed that there was higher presence of Aggregatibacter actinomycetemcomitans, Prevotella nigrescens, and $P$. intermedia were associated with PI biofilms [11]. As with periodontal disease, poor hygiene and the absence of a maintenance phase are considered the most common risk factors for PI. It has been reported that people with poor oral hygiene and a smoking habit have three times greater probability of suffering PI [12].

Periodontal disease has been identified as the major risk factor for developing PI and thus is negative indicator for implant survival. In cohort studies, systematic reviews and meta-analyses have reported that the prevalence for PI is 2.29 greater in patients with periodontal disease [13]. Other studies have also shown that patients with the presence of periodontal pockets $\geq 6 \mathrm{~mm}$ have an increased risk of developing PI [14]. Fürst reported in a clinical study that in early biofilm colonization patterns between tooth and one-stage implants bacterial colonization happens within $30 \mathrm{~min}$ after dental implant placement. Additionally, the early colonization patterns differed between implant and tooth surfaces, and it is important to mention that in this study, patients never had periodontal disease [15].

We could say that PI is a bacterial condition, but also, we must remember that it occurs in a specific site, unlike periodontitis, in which various areas of the mouth are usually affected; therefore, it is logical to think that several systemic and local factors could influence the appearance of PI before it actually appears.

As examples, factors, such as poor oral hygiene, smoking, and/or hyperglycemia, it has been shown that once PI appears, it follows a non-linear and accelerated pattern, but it can be asked, "what causes a mucositis to take turns in periimplantitis?".

The answer lies in the activation of epigenetic factors that cause non-expression of genes involved in bone matrix synthesis; therefore, suppressing osteogenesis and on the other hand, over-expression of genes also activates osteoclastogenesis [9]. Thus, we seek to establish that it is not only bacterial etiology of PI disease but local and systemic factors that may be present even before the homeostasis between health and PI disease breaks down.

PI and periodontal disease are characterized by soft tissue inflammation and bone destruction. In these conditions, chemically reactive oxygen species (ROS) have a significant function in cell signaling, maintenance, and proliferation in the periodontal tissues in response to different bacteria [16]. ROS accumulation leads to oxidative stress, which is responsible for cell damage and periodontal destruction. Several studies have shown that free radicals, ROS, and OS are involved in oral diseases, especially in periodontal diseases; however, their role in PI remains unclear [16],[17], [18]. 


\section{OXIDATIVE STRESS (OS) AND PERI-IMPLANTITIS}

The term of OS is applied when the antioxidant balance is shifted toward pro-oxidant agents due to an increment in ROS when levels of antioxidants are diminished. OS is related to damaged biomolecules, such as carbohydrates, lipids, proteins, and nucleic acids and can promote cell death. OS leads to the generation of ROS and free radicals and has been identified as clinically relevant in vascular, inflammatory, and periodontal diseases [19]-[23].

\section{FREE RADICALS}

ROS, such as products of cellular metabolism in organisms, are regulated by a system of antioxidant defense. ROS can be classified as two types: (1) free radicals (superoxide radical and the hydroxyl radical and (2) nonradical oxygen species (hydrogen peroxide, the singlet oxygen, peroxynitrite [24]-[26]. Free radicals are unstable atoms and molecules with incomplete electron shells capable of inducing structural and functionals changes in biomolecules, which can result in cell damage. Inflammation and environmental exposures, such as tobacco smoke and radiation, lead to higher production of this molecular species.

During inflammatory processes, macrophages generate an extensive amount free radical causing damage of most biomolecules (lipids, carbohydrates, proteins, and nucleic acids) and also oxidative enzymes. Free radicals also increase pro-inflammatory cytokines, such as tumor necrosis factor (TNF)- $\alpha$, interleukins (IL1- $\beta$ and 6) [26].

One of the main targets of free radicals are cell membrane polyunsaturated fatty acids in which free radicals promote lipid peroxidation [28], [27]. This process generates numerous sub-products, such as malondialdehyde (MDA), which is a highly toxic molecule that disrupts the cell membrane and leads to cell damage and an increase in OS. MDA is used to evaluate OS levels by measuring in tissues, gingival, and peri-implant crevicular fluid [16]. Studies have reported that levels of MDA are augmented in oral fluids derived from saliva and gingival crevicular from individuals with periodontal and PI disease [18], [26], [34].

Another biological biomarker of oxidative stress is myeloperoxidase (MPO), which is an inflammatory and oxidative enzyme with antimicrobial activity that catalyzes the transformation of hydrogen peroxide $\left(\mathrm{H}_{2} \mathrm{O}_{2}\right)$ to hypochlorous acid in neutrophils. MPO is a potential oxidizing agent involved host defense against microorganisms [17].

MPO plays a relevant role in inflammatory disease processes [30]. It has been reported in the literature that MPO activity is associated with periodontal tissue inflammation, and MPO levels also increases in saliva and crevicular fluid at the surrounding tissues of dental implants that are associated with peri-implant disease [18], [31].

ROS promotes an increase in the products of nonenzymatic glycation, several studies report that the end products of advanced glycation (AGEs) induce proinflammatory cytokines (TNF- $\alpha$, IL6 and 8) and OS [32], [33]. Ramzan and Malik reported the presence of higher levels of AGEs in subjects with PI and implant failure within the first six months after implantation in which the number of
AGEs was similar to those of chronic periodontitis patients [34], [35].

There are reports that free radicals, ROS, MDA, AGEs, and MPO levels are elevated in oral fluids in patients with periodontitis and PI diseases and can promote inflammation and periodontal tissue damage [18]. In future research, it is important to analyze the presence of ROS, MDA, and MPO in saliva or gingival crevicular fluid to differentiate between healthy and PI implants [36], [37].

\section{OXIDATIVE STRESS, DENTAL IMPLANT PlacEMENT AND OSSEOINTEGRATION}

Osseointegration is a term that describe an organizational and operative relationship with the bone tissues and the implant surface [39]. Dental implant materials are usually made of titanium (Ti) or Ti alloy. When this material makes contact with the oxygen a layer is created of titanium oxide $\left(\mathrm{TiO}_{2}\right)$ at the dental implant surface and their integrity is important to evade problems at the bone-implant connection (osseointegration) and prevent their corrosion [39]. After the dental implant placement, structural changes can occur, such as compression and microfractures in the dental implant surface area. Torsional and frictional forces may alter the original implant surface thus damaging the oxide layer recently created at the implant. Stress areas can show Ti fragments that are related to the generation of inflammation and lead to an increase interleukins and cytokines, including TNF- $\alpha$, IL1- $\beta$, and receptor activator of nuclear factor kappa$B$ ligand (RANKL). Stress areas show alterations in epithelial cells with an increase in DNA damage response [39].

After placement of a dental implant, an inflammatory healing response occurs. Angiogenesis is one characteristic of the process and it contributes cells and oxygen that are important for the achievement of osseointegration. Tsarik et al. analyzed the damage to endothelial cells and found cytotoxicity and inhibition of cell proliferation when cathodic corrosion of Ti was induced. They observed that ROS and $\mathrm{H}_{2} \mathrm{O}_{2}$ were sub-products of the corrosion process and that these particles harm endothelial cells. If dysregulation in the inflammatory process and accumulation of ROS particles over the implant and surrounding tissues occurs, aseptic loosening of the implant may also occur [40] (Fig. 2-A).

\section{THE TRIBOCORROSION OF DENTAL IMPLANTS AND ITS RELATIONSHIP WITH PERI-IMPLANTITIS}

Tribocorrosion refers to affectation of a material, in this case titanium-alloy, as a result of concurrent actions by chemical, mechanical (wear), and electromechanical (corrosion) factors. When this damaging process happens in biological environments, such as the human mouth, the process is called bio-tribocorrosion [43]. The formation process of bacterial biofilm begins with the union of primary bacteria adhesins to the layer of glycoproteins found over the surface of soft (gums) and hard (enamel) tissues or restorative structures (implants). Later, primary bacteria (Streptococcus. mutans, sanguinis, oralis, gordonii), Actinomyces naeslundii, Fusobacterium nucleatum, and Streptococcus mitis) adhesion to the layer of glycoproteins (mucin) [44], bacteria begin the 
formation of a matrix composed by water, nucleic acids, glycoproteins and polysaccharides [41], [45]. When oxygen levels decrease, phenotypical changes occur to the bacterial colony, and a periodontopathogen biofilm is formed. These late bacterial colonizers are an anaerobic lineage that can produce endotoxins. As a result of carbohydrate digestion by the biofilm bacteria, lactic acid is produced, and the resulting changes in the $\mathrm{pH}$ environment can cause alteration of the layer of $\mathrm{TiO}_{2}$ with later corrosion of the dental implant. In in vitro experimental studies, Bressan et. al reported that $\mathrm{Ti}$ particles could affect mitochondrial functions and promote ROS production in peri-implant tissues [46].

ROS molecules generate an inflammatory response and destruction of the tissues surrounding a dental implant. Also, endotoxins produced by anaerobic bacteria and $\mathrm{Ti}$ ions transport into tissues occur as a results of implant corrosion. This series of events establishes a retro-negative cycle that modifies the homeostasis between the surrounding tissues of dental implants and their surfaces. (Fig. 2-B).

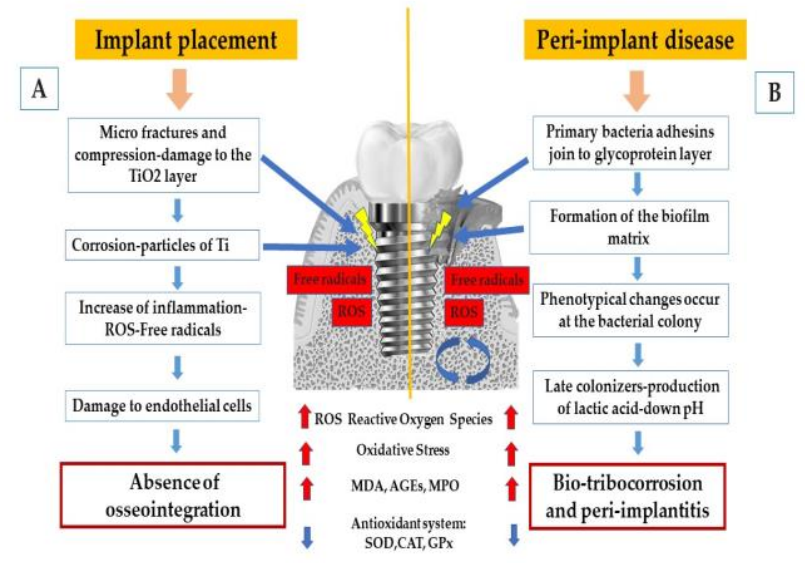

Fig. 2. A-B). Fig 2. Role of oxidative stress (OS) during dental implant placement and their influence on peri-implantitis.

After dental implant placement, modifications can occur at the implant surfaces and its surrounding bone; also, other forces may alter the dental implant surface thus damaging the layer of $\mathrm{TiO} 2$ [38], which may lead to $\mathrm{Ti}$ corrosion and the increment of free radicals due to oxidative stress (OS). Damage to the endothelial cells can occur, thus leading to loss of the dental implant.

In PI disease, pathogen biofilm mechanisms that produce lactic acid by the metabolism of carbohydrates are involved. This causes a decrease in $\mathrm{pH}$ at the zone, and biotribocorrosion may occur leading to a retro-negative cycle that will affect the implant.

\section{ANTIOXIDANTS AND THEIR ROLE IN PERI-IMPLANTITIS}

The term, antioxidant, is defined as a substance that protects or prevent cells and DNA from the damage induced by free radicals, which are converted into waste products that are eliminated from the human body. Antioxidants may play a role in chronic and systemic diseases, they can end oxidative reactions to avoid new oxidation reactions, the inability of human body to neutralize large amounts of free radicals to which we are exposed allows the presence of diseases, including oral diseases, and thus, it is necessary to consume natural antioxidants in the diet that are capable of neutralizing free radicals [50]-[52]. In oral diseases, such as periodontal and PI, saliva is important in the oral cavity for maintaining homeostasis. The salivary glands produce saliva, which represents a defense system with enzymes to prevent or block the generation of superoxide dismutase (SOD), glutathione peroxidase (GPx), glutathione reductase, catalase (CAT), and free radicals [20].

One study analyzed total antioxidant capacity of saliva and found that it was reduced in people with PI [18]. Other studies have reported that antioxidants decrease the presence of the enzymes, SOD, CAT, and GPX, and further increase MDA activity in periodontal disease and PI [55]. Several experimental studies have reported the benefits of antioxidants in inflammatory processes. Another study reported that antioxidants have protective effects on the periodontium, and they neutralize ROS to prevent tissue damage [54].

Antioxidants may play a role to prevent or control PI. In an experimental study by Ozawa et al., novel therapeutic strategy with a hydrogel containing nitroxide radicals was injected in an experimental rat model with PI and osteoblast cells in vitro was used. The hydrogel reduces the presence of ROS and inhibits $\mathrm{H} 2 \mathrm{O} 2$ and lipid peroxidation in vitro. This gel could be a prophylactic therapy for causing a decrease in inflammatory responses and the effects associated with PI [53]. However, the role of OS and antioxidant capacity in PI requires more experimental and clinical studies.

\section{CLINICAL SugGESTIONS}

Once we have presented the possible mechanisms by which there could be an absence of osseointegration and a PI disease due to inflammation and structural changes on the implant surface, we can provide some critical suggestions in order to prevent such events. We describe an approach comprised by two components: (1) prevention and (2) treatment.

\section{A. At the Time of Implant Dental Placement}

Enough keratinized tissue in the area of the placement must exist. It has been proven that having a good band of keratinized tissue around dental implants positively impacts them by preventing recession, accumulation of plaque, and deepening of the vestibule. These conditions provide an optimal environment for hygiene. These characteristics have been correlated with immune responses in which molecules such as prostaglandin E2 (PGE2) are not present [9].

In the surgery involving dental implant placement, their surfaces could take contact with the mucous tissues or saliva, this must be avoided in order to decrease the contamination of the implant; this will avoid the presence of bacteria and prevent the damage in the recently formed $\mathrm{TiO}_{2}$ layer [56]. In an in vitro study, Shams et al. analyzed the effects of saliva on morphology and differentiation of osteoblast cells attached to implant contaminated surfaces and identified that cells in the experimental group were round with shorter cellular processes, whereas elongated, fusiform, and well-defined cell processes were seen in the control condition (not exposed to saliva). Also, alkaline phosphatase activity was lower in the test group. This finding indicates the importance of not 
allowing contamination of the implant surface or the operative field [57].

Excessive torques that cause disruption of the $\mathrm{TiO}_{2}$ layer with subsequent corrosion at level of the dental implant and release of $\mathrm{Ti}$ particles to the tissues surrounding a dental implant must be avoided [38]. As we mentioned above, this process can cause uncontrolled inflammation that could damage the endothelial cells and therefore, affect the viability of the peri-implant tissues, resulting in a lack of osseointegration. In the areas in which the implant cords contact the bone, there is reabsorption in the first weeks; however, in contrast, those places in which no contact is found, osteoblastic differentiation and therefore bone formation is initiated [9].

Vestibular bone mostly consists of cortical bone, and it receives irrigation from the periosteum on the outer side and from endosteum on the inner side. Placement of dental implants in a very vestibular position compromises the endosteal circulation and with it, the vestibular bone (Fig. 1). Thickness of the vestibular and lingual bones in the areas in which dental implants are located, a minimum of $1.5 \mathrm{~mm}$ is needed to avoid the bone remodeling phenomena due to avascular necrosis caused by improper positioning of the implants.

Regarding the apico-coronal positions of the implants when they are placed $>6 \mathrm{~mm}$ below the cement-enamel junctions of the adjacent teeth, they present a higher incidence of PI [9].

Mesio-distal positions of the implants must consider the distance between them and between teeth as this will allow adequate spaces, so hygiene can be performed around them. This process also prevents necrosis of interproximal bone due the proximity of the structures [9].

Immediate loading of the implants must be done following the various consensus protocols that have already been established. This text contains information about the characteristics that an implant and its surrounding tissues must have at the time of its placement in order to be loaded [58].

The fact of performing immediate loads over implants that do not have these parameters (minimum torque, primary stability) can lead to a high risk of dental implant failure.

\section{B. At the Time of Maintenance Phase}

\section{a) Prevention of problems on loaded implants}

Supportive peri-implant therapy and periodontal healthcare should be undertaken every three, six, or 12 months after agreeing to the specific needs of the patient. Detection of problems in their early stages ensures that a less invasive treatment would be performed. In patients with a history of periodontitis, shorter recall periods should be considered. It is necessary that oral hygiene education be assessed and evaluated with the patient to maintain periimplant health [59].

The clinical examination at the recall visits must include a physical examination: (1) inspection and (2) palpation of the peri-implant tissues to analyze if there is presence of swelling, bleeding, and suppuration. Radiography is recommended to evaluate crestal bone level, and comparison with previous radiographs are suggested to analyze progressive bone loss [60].
It is recommended that measurements of probe depth in implants be performed without exerting too much pressure in order to avoid disruption at the mucosa-implant union, preferably using a compatible probe to prevent scratching of the dental implant surface.

Scaling procedures will also occur during the recall appointments in order to reduce the risk of suffering PI. Using plastic curette to removing biofilm is not recommended as it can also leave traces of plastic particles between the gum and the implant due to implant hardness. For the appropriate removal of biofilm and calculus, metal scalers and curettes compatible with Ti must be used. A need for research regarding the most effective tool for systematic and professional mechanical debridement for dental implants is required [61].

\section{b) Treatment of PI diseases in loaded implants}

The most common problems found in this phase are PM and PI; treatment of the latter could be surgical or nonsurgical to remove biofilm and calculus, a process that would result in the resolution of inflammation around implant tissues.

Several instruments have been proposed to treat these pathological condition, including the use of scaling instruments, implantoplasty, air abrasion, chemical, and laser decontamination methods. Depending on the particular situation, the clinician should adopt the treatment modality that results in less liberation of Ti particles to the surrounding tissues (Fig. 3).

Implantoplasty could be the most invasive treatment against PI. Powerful suction and high irrigation must be used in this method because it has been proven that it causes a large number of $\mathrm{Ti}$ particles can gain access to the surrounding tissues, thus causing inflammation [38].

In a study by Ronay et al., three debridement techniques using different instruments, such as scalers, curettes, airpowder abrasion, and ultrasonic were evaluated. They concluded that air-powder abrasion has more advantages, such as no effects on the Ti surface, over the other methods [62].

With respect to lasers, it is not recommended to use them for long periods. Avoiding over-irradiation is also important and can be done by using a refrigerant media, such as water or air. The laser must be configured in the pulse mode at low energies [38].

Several chemical decontamination methods for treating PI have been tested. These methods include tetracycline, doxycycline, chlorhexidine, hydrogen peroxide, and citric acid. High concentrations of fluoride and acid solutions alter the TiO2 layer, so their use must be avoided. Solutions that have shown better behavior with the smallest modification to the dental implant surfaces are chlorhexidine, hydrogen peroxide, and saturated citric acid [38]. 


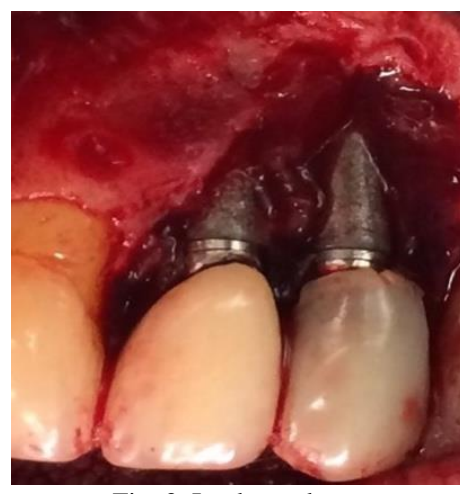

Fig. 3. Implantoplasty.

When the management of PI implies a surgical approach, a technique depending on the final objective of the surgery should be chosen. In a systemic review by Esposito et al. several suggestions have been made: (1) open flap debridement in order to remove bacteria and unsupported implant threads that protects bacterial biofilm, (2) smoothing of the dental implant surface to reduce the surface roughness, and (3) decontamination or detoxification using several chemical agents or laser [63], [64] (Fig. 4).
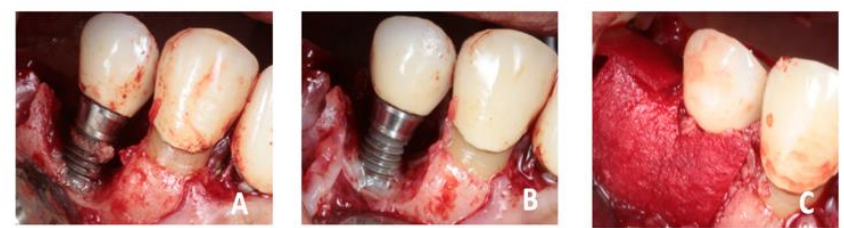

Fig. 4. Case 1. A-B) Open flaps to provide access to the diseased areas in order to treat them by the several decontamination methods. C) Guided bone regeneration using a resorbable membrane in which regenerative techniques, such as guided bone regeneration and autologous or allogenic bone grafts, are used.

\section{CONCLUSION}

Oxidative stress can play a role in PI, and the use of antioxidants to prevent a lack of osseointegration or PI requires additional studies in order to prevent dental implant complications and failures.

Lactic acid production by several microorganisms in PI causing dental implant surface corrosion, and other factors, such as periodontitis, smoking, and overload can also contribute to inducing and supporting bone destruction around dental implants.

The placement of a dental implant occurs in several stages: (1) insertion, (2) loading, and (3) maintenance. Damage to the $\mathrm{TiO} 2$ layer can cause corrosion that may lead to liberation of $\mathrm{Ti}$ particles into the surrounding tissues and produced inflammation, and it may contribute to a lack of osseointegration, PI, damage, and/or contamination of the surrounding tissues.

Clinicians must be careful at every stage of implant placement, analyze each case individually, and make decisions based on scientific literature in order to prevent improper treatment and tissue damage. A thorough clinical history of the patient's oral health record should be performed, and key risk factors that may affect the osseointegration process at any stage should be examined.

\section{ACKNOWLEDGMENT}

The authors special thanks to Dr. Andres Peñalva Tron and Dr. Marco Antonio Iturbe Medina from Periodontics Department of Intercontinental University to get the clinical photos of PI.

\section{REFERENCES}

[1] Turkyilmaz, I.; Tozum, T.F. 30-Year Outcomes of Dental Implants Supporting Mandibular Fixed Dental Prostheses: A Retrospective Review of 4 Cases. Implant Dent 2015, 24, 620-624.

[2] Caton, J.G.; Armitage, G.; Berglundh, T.; Chapple, I.L.C.; Jepsen, S.; Kornman, K.S.; Mealey, B.L.; Papapanou, P.N.; Sanz, M.; Tonetti, M.S. A new classification scheme for periodontal and peri-implant diseases and conditions - Introduction and key changes from the 1999 classification. Journal of Clinical Periodontology 2018, 45, S1-S8, doi:10.1111/jcpe. 12935 .

[3] A. Mombelli, M. A. van Oosten, E. Schurch Jr, N. P. Land. The Microbiota Associated With Successful or Failing Osseointegrated Titanium Implants. Oral Microbiol Immunol. 1987 Dec;2(4):145-51

[4] Academy Report: PM and Peri-implantitis: A Current Understanding of Their Diagnoses and Clinical Implications. Journal of Periodontology 2013, 84, 436-443, doi:10.1902/jop.2013.134001.

[5] E. Jung, R.; Zembic, A.; Pjetursson, B.E.; Zwahlen, M.; S. Thoma, D. Systematic review of the survival rate and the incidence of biological, technical, and aesthetic complications of single crowns on implants reported in longitudinal studies with a mean follow-up of 5 years. Clinical Oral Implants Research 2012, 23, 2-21, doi:10.1111/j.16000501.2012.02547.x.

[6] H. Dreyer, J. Grischke, C. Tiede, J. Eberhard, A. Schweitzer , S. E. Toikkanen, S. Glöckner, G. Krause, M. Stiesch. Epidemiology and Risk Factors of Peri-implantitis: A Systematic Review. J Periodontal Res. 2018 Oct;53(5):657-681.

[7] Mischa Krebs, Nikolina Kesar, Amira Begić, Nadine von Krockow, Georg-Hubertus Nentwig, Paul Weigl. Incidence and Prevalence of Peri-implantitis and PM 17 to 23 (18.9) Years Postimplant Placement. Clin Implant Dent Relat Res. 2019 Dec;21(6):1116-1123

[8] Johannes Krisam, Larissa Ott, Stephanie Schmitz, Anna-Luisa Klotz, Aida Seyidaliyeva, Peter Rammelsberg, and Andreas Zenthöfer. Factors affecting the early failure of implants placed in a dental practice with a specialization in implantology-a retrospective study. BMC Oral Health (2019) 19:208.

[9] Alberto Monje, Angel Insua and Hom-Lay Wang. Understanding periimplantitis as a Plaque-Associated and Site-Specific Entity: On the Local Predisposing Factors. Journal of clinical medicine. 2019.

[10] Schwarz, F.; Derks, J.; Monje, A.; Wang, H.-L. Peri-implantitis. Journal of Periodontology. 2018, 89, S267-S290, doi:10.1002/jper.160350 .

[11] Sahrmann, P.; Gilli, F.; Wiedemeier, D.B.; Attin, T.; Schmidlin, P.R.; Karygianni, L. The Microbiome of peri-implantitis: A Systematic Review and Meta-Analysis. Microorganisms 2020, 8, 661.

[12] Lin, C.Y.; Chen, Z.; Pan, W.L.; Wang, H.L. Is History of Periodontal Disease Still a Negative Risk Indicator for Peri-implant Health Under Supportive Post-implant Treatment Coverage? A Systematic Review and Meta-analysis. Int J Oral Maxillofac Implants 2020, 35, 52-62, doi:10.11607/jomi.7714.

[13] Ferreira SD, Martins CC, Amaral SA, Vieira TR, Albuquerque BN, Cota LOM, et al. Periodontitis as a risk factor for peri-implantitis: Systematic review and meta-analysis of observational studies. J Dent. 2018;79:1-10.

[14] Cho-Yan Lee J, Mattheos N, Nixon KC, Ivanovski S. Residual periodontal pockets are a risk indicator for peri-implantitis in patients treated for periodontitis. Clin Oral Implants Res. 2012;23(3):325-33.

[15] Furst, M.M.; Salvi, G.E.; Lang, N.P.; Persson, G.R. Bacterial colonization immediately after installation on oral titanium implants. Clin Oral Implants Res 2007, 18, 501-508, doi:10.1111/j.16000501.2007.01381.x.

[16] Mahvash Mousavi Jazi, H.R.S.P.R., Faezeh Mirmiran. Level of Oxidative Stress Markers in Peri-Implant Crevicular Fluid and Their Corre. Journal of Dentistry 2015, 12, 340-346.

[17] Sanchez-Siles, M.; Lucas-Azorin, J.; Salazar-Sanchez, N.; CarbonellMeseguer, L.; Camacho-Alonso, F. Salivary Concentration of Oxidative Stress Biomarkers in a Group of Patients with PI: A Transversal Study. Clin Implant Dent Relat Res 2016, 18, 1015-1022, doi:10.1111/cid.12367.

[18] Liskmann, S.; Vihalemm, T.; Salum, O.; Zilmer, K.; Fischer, K.; Zilmer, M. Characterization of the antioxidant profile of human saliva 
in peri-implant health and disease. Clinical Oral Implants Research 2007, 18, 27-33, doi:10.1111/j.1600-0501.2006.01296.x.

[19] Tothova, L.; Celec, P. Oxidative Stress and Antioxidants in the Diagnosis and Therapy of Periodontitis. Front Physiol 2017, 8, 1055 doi:10.3389/fphys.2017.01055.

[20] Zukowski, P.; Maciejczyk, M.; Waszkiel, D. Sources of free radicals and oxidative stress in the oral cavity. Arch Oral Biol 2018, 92, 8-17, doi:10.1016/j.archoralbio.2018.04.018.

[21] Gayosso-Islas, E.; Snchez- Moreno, C.; Valadez-Vega, C.; MoralesGonzlez, n.; Esquivel Soto, J.; Esquivel-Chirino, C.; Garca-Luna, M.; Madrigal-Santill, E. Protective Effect of Silymarin on Liver Damage by Xenobiotics. In Oxidative Stress and Chronic Degenerative Diseases - A Role for Antioxidants, 2013; 10.5772/51502.

[22] Gutteridge, J.M.C.; Halliwell, B. Mini-Review: Oxidative stress, redox stress or redox success? Biochem Biophys Res Commun 2018, 502, 183-186, doi:10.1016/j.bbrc.2018.05.045.

[23] Valko, M.; Leibfritz, D.; Moncol, J.; Cronin, M.T.; Mazur, M.; Telser, $\mathrm{J}$. Free radicals and antioxidants in normal physiological functions and human disease. Int $J$ Biochem Cell Biol 2007, 39, 44-84, doi:10.1016/j.biocel.2006.07.001.

[24] Fernandez-Sanchez, A.; Madrigal-Santillan, E.; Bautista, M.; EsquivelSoto, J.; Morales-Gonzalez, A.; Esquivel-Chirino, C.; DuranteMontiel, I.; Sanchez-Rivera, G.; Valadez-Vega, C.; Morales-Gonzalez, J.A. Inflammation, oxidative stress, and obesity. Int J Mol Sci 2011, 12, 3117-3132, doi:10.3390/ijms12053117.

[25] Tunez, I.; Feijoo, M.; Huerta, G.; Montilla, P.; Munoz, E.; Ruiz, A.; Collantes, E. The effect of infliximab on oxidative stress in chronic inflammatory joint disease. Curr Med Res Opin 2007, 23, 1259-1267, doi:10.1185/030079907X187955.

[26] Trivedi, S.; Lal, N. Antioxidant enzymes in periodontitis. J Oral Biol Craniofac Res 2017, 7, 54-57, doi:10.1016/j.jobcr.2016.08.001.

[27] Nazaryan, R.; Kryvenko, L. Salivary oxidative analysis and periodontal status in children with atopy. Interv Med Appl Sci 2017, 9, 199-203, doi:10.1556/1646.9.2017.32

[28] Morales-Gonzalez, J.A.; Gutierrez-Salinas, J.; Garcia-Ortiz, L.; De Carmen Chima-Galan, M.; Madrigal-Santillan, E.; Esquivel-Soto, J.; Esquivel-Chirino, C.; Gonzalez-Rubio, M.G. Effect of sodium fluoride ingestion on malondialdehyde concentration and the activity of antioxidant enzymes in rat erythrocytes. Int J Mol Sci 2010, 11, 24432452, doi:10.3390/ijms11062443.

[29] Chapple, I.L.C. Role of free radicals and antioxidants in the pathogenesis of the inflammatory periodontal diseases. J Clin Pathol: Mol Pathol 1996, 49, 247-255.

[30] Van der Veen, B.S.; de Winther, M.P.; Heeringa, P. Myeloperoxidase: molecular mechanisms of action and their relevance to human health and disease. Antioxid Redox Signal 2009, 11, 2899-2937, doi:10.1089/ars.2009.2538.

[31] Yamalik, N.; Çağlayan, F.; Kilinç, K.; Kilinç, A.; Tümer, C. The Importance of Data Presentation Regarding Gingival Crevicular Fluid Myeloperoxidase and Elastase-Like Activity in Periodontal Disease and Health Status. Journal of Periodontology 2000, 71, 460-467, doi:10.1902/jop.2000.71.3.460.

[32] Topkas, E.; Keith, P.; Dimeski, G.; Cooper-White, J.; Punyadeera, C. Evaluation of saliva collection devices for the analysis of proteins. Clin Chim Acta 2012, 413, 1066-1070, doi:10.1016/j.cca.2012.02.020.

[33] Guo, M.; Liu, L.; Zhang, J.; Liu, M. Role of Reactive Oxygen Species and Advanced Glycation End Products in the Malfunctioning of Denta Implants. West Indian Med J 2015, 64, 419-423, doi:10.7727/wimj.2014.105.

[34] Ramzan, H.s. Role Of Advanced Glycation End Products (Ages) And Oxidative Stress In The Failure Of Dental Implants. Dentistry 2013, 04, doi:10.4172/2161-1122.1000179.

[35] Pietropaoli, D.; Monaco, A.; Del Pinto, R.; Cifone, M.G.; Marzo, G.; Giannoni, M. Advanced glycation end products: possible link between metabolic syndrome and periodontal diseases. Int J Immunopathol Pharmacol 2012, 25, 9-17, doi:10.1177/039463201202500102.

[36] Gomes, A.M.; Douglas-de-Oliveira, D.W.; Oliveira Costa, F. Could the biomarker levels in saliva help distinguish between healthy implants and implants with peri-implant disease? A systematic review. Arch Oral Biol 2018, 96, 216-222, doi:10.1016/j.archoralbio.2018.09.008.

[37] Ghallab, N.A. Diagnostic potential and future directions of biomarkers in gingival crevicular fluid and saliva of periodontal diseases: Review of the current evidence. Archives of Oral Biology 2018, 87, 115-124, doi:https://doi.org/10.1016/j.archoralbio.2017.12.022.

[38] Delgado-Ruiz, R.; Romanos, G. Potential Causes of Titanium Particle and Ion Release in Implant Dentistry: A Systematic Review. Int J Mol Sci 2018, 19, doi:10.3390/ijms19113585.

[39] Suarez-Lopez Del Amo, F.; Garaicoa-Pazmino, C.; Fretwurst, T. Castilho, R.M.; Squarize, C.H. Dental implants-associated release of titanium particles: A systematic review. Clin Oral Implants Res 2018 , 10.1111/clr.13372

[40] Tsaryk, R.; Kalbacova, M.; Hempel, U.; Scharnweber, D.; Unger, R.E.; Dieter, P.; Kirkpatrick, C.J.; Peters, K. Response of human endothelial cells to oxidative stress on Ti6Al4V alloy. Biomaterials 2007, 28, 806813, doi:10.1016/j.biomaterials.2006.09.033

[41] Souza, J.C.; Henriques, M.; Oliveira, R.; Teughels, W.; Celis, J.P.; Rocha, L.A. Biofilms inducing ultra-low friction on titanium. J Dent Res 2010, 89, 1470-1475, doi:10.1177/0022034510378428.

[42] Yan, Y.; Neville, A.; Dowson, D. Biotribocorrosion - an appraisal of the time dependence of wear and corrosion interactions: I. The role of corrosion. Journal of Physics D: Applied Physics 2006, 39, 3200-3205, doi:10.1088/0022-3727/39/15/s10.

[43] Affatato, S.; Grillini, L. 1 - Topography in bio-tribocorrosion. In BioTribocorrosion in Biomaterials and Medical Implants, Yan, Y., Ed. Woodhead Publishing: 2013, https://doi.org/10.1533/9780857098603.1pp. 1-22a.

[44] Ge, J.; Catt, D.M.; Gregory, R.L. Streptococcus mutans surface alphaenolase binds salivary mucin MG2 and human plasminogen. Infect Immun 2004, 72, 6748-6752, doi:10.1128/IAI.72.11.6748-6752.2004.

[45] Vinogradov, A.M.; Winston, M.; Rupp, C.J.; Stoodley, P. Rheology of biofilms formed from the dental plaque pathogen Streptococcus mutans. Biofilms 2004, 1, 49-56, doi:10.1017/s1479050503001078.

[46] Schwartz-Arad, D.; Mijiritsky, E.; Penarrocha, M., et al. Metal Nanoparticles Released from Dental Implant Surfaces: Potentia Contribution to Chronic Inflammation and Peri Implant Bone Loss. Materials (Basel) 2019, 12, doi:10.3390/ma12122036.

[47] Ratner, B.D.; Hoffman, A.S.; Schoen, F.J.; Lemons, J.E. Introduction Biomaterials Science: An Evolving, Multidisciplinary Endeavor. In Biomaterials Science (Third Edition), Ratner, B.D., Hoffman, A.S. Schoen, F.J., Lemons, J.E., Eds. Academic Press: 2013 https://doi.org/10.1016/B978-0-08-087780-8.00153-4pp. Xxv-Xxxix. pp 267-271.

[48] Guindy, J.S.; Schiel, H.; Schmidli, F.; Wirz, J. Corrosion at the marginal gap of implant-supported suprastructures and implant failure. Int J Oral Maxillofac Implants 2004, 19, 826-831.

[49] Mabilleau, G.; Bourdon, S.; Joly-Guillou, M.L.; Filmon, R.; Basle, M.F.; Chappard, D. Influence of fluoride, hydrogen peroxide and lactic acid on the corrosion resistance of commercially pure titanium. Actc Biomater 2006, 2, 121-129, doi:10.1016/j.actbio.2005.09.004.

[50] Esquivel-Chirino, C.; Esquivel-Soto, J.; Antonio, J.; Montes, D.; Luis, J.; Hernndez-Mora, L.E.; Zentella-Dehes, A. Inflammatory Environmental, Oxidative Stress in Tumoral Progression. In Oxidative Stress and Chronic Degenerative Diseases - A Role for Antioxidants, 2013; 10.5772/51789.

[51] Vargas-Mendoza, N.; Madrigal-Santillan, E.; Morales-Gonzalez, A.; Esquivel-Soto, J.; Esquivel-Chirino, C.; Garcia-Luna, Y.G.-R.M.; Gayosso-de-Lucio, J.A.; Morales-Gonzalez, J.A. Hepatoprotective effect of silymarin. World J Hepatol 2014, 6, 144-149, doi:10.4254/wjh.v6.i3.144.

[52] Madrigal-Santillan, E.; Fragoso-Antonio, S.; Valadez-Vega, C.; Solano-Solano, G.; Perez, C.Z.; Sanchez-Gutierrez, M.; IzquierdoVega, J.A.; Gutierrez-Salinas, J.; Esquivel-Soto, J.; Esquivel-Chirino C., et al. Investigation on the protective effects of cranberry against the DNA damage induced by benzo[a]pyrene. Molecules 2012, 17, 44354451, doi:10.3390/molecules17044435.

[53] Ozawa, R.; Saita, M.; Sakaue, S.; Okada, R.; Sato, T.; Kawamata, R.; Sakurai, T.; Hamada, N.; Kimoto, K.; Nagasaki, Y. Redox injectable gel protects osteoblastic function against oxidative stress and suppresses alveolar bone loss in a rat peri-implantitis model. Acta Biomaterialia 2020. doi:https://doi.org/10.1016/j.actbio.2020.04.003.

[54] Kaur, G.; Kathariya, R.; Bansal, S.; Singh, A.; Shahakar, D. Dietary antioxidants and their indispensable role in periodontal health. J Food Drug Anal 2016, 24, 239-246, doi:10.1016/j.jfda.2015.11.003.

[55] Sobaniec, S.-L. Morphological examinations of hard tissues of paradontium and evaluation of selected processes of 2000.

[56] Jinno, Y.; Toia, M.; Stocchero, M.; Jimbo, R.; Stavropoulos, A.; Becktor, J.P. Effects of saliva contamination on osseointegration during dental implant surgery in augmented areas. Clinical Oral Implants Research 2018, 29, 224-224, doi:10.1111/clr.109_13358.

[57] Shams, N.; Ghasemi, M.; Sadatmansouri, S.; Bonakdar, S. Morphology and Differentiation of MG63 Osteoblast Cells on Saliva Contaminated Implant Surfaces. J Dent (Tehran) 2015, 12, 424-429.

[58] Gallucci, G.O.; Hamilton, A.; Zhou, W.; Buser, D.; Chen, S. Implan placement and loading protocols in partially edentulous patients: A systematic review. Clinical Oral Implants Research 2018, 29, 106-134, doi:10.1111/clr.13276

[59] Jepsen, S.; Berglundh, T.; Genco, R.; Aass, A.M.; Demirel, K.; Derks J.; Figuero, E.; Giovannoli, J.L.; Goldstein, M.; Lambert, F., et al Primary prevention of peri-implantitis: Managing PM. Journal of 
Clinical Periodontology 2015, 42, S152-S157, doi:10.1111/jcpe.12369.

[60] Lang, N.P.; Adler, R.; Joss, A.; Nyman, S. Absence of bleeding on probing An indicator of periodontal stability. Journal of Clinical Periodontology $1990, \quad 17, \quad 714-721, \quad$ doi:10.1111/j.1600051X.1990.tb01059.x.

[61] Clark, D.; Levin, L. Dental implant management and maintenance: How to improve long-term implant success? Quintessence Int 2016, 47, 417-423, doi:10.3290/j.qi.a35870.

[62] Ronay, V.; Merlini, A.; Attin, T.; Schmidlin, P.R.; Sahrmann, P. In vitro cleaning potential of three implant debridement methods. Simulation of the non-surgical approach. Clinical Oral Implants Research 2017, 28, 151-155, doi:10.1111/clr.12773.

[63] Figuero, E.; Graziani, F.; Sanz, I.; Herrera, D.; Sanz, M. Management of PM and peri-implantitis. Periodontology 2000 2014, 66, 255-273, doi:10.1111/prd.12049.

[64] Esposito, M.; Grusovin, M.G.; Worthington, H.V. Treatment of Periimplantitis: what interventions are effective? A Cochrane systematic review. Int J Oral Implantol 2012, 5, 21-41.

[65] Tomasi Regidor-Vigón Derks Cristiano, Erik Alberto Ortiz- Jan. Efficacy of reconstructive surgical therapy at peri-implantitis-related bone defects. A systematic review and meta-analysis. Journal of clinical periodontology. 2018.

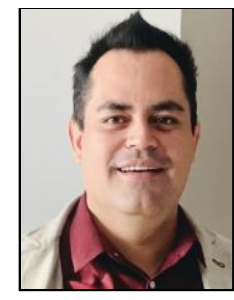

C. Esquivel-Chirino*

$\mathrm{PhD}$ in Oral Biology

Master of Sciences in Oral Biology

Dentist

Dental Researcher

Professor 